\section{Fatores de risco para mortalidade neonatal em Blumenau, Santa Catarina: linkage entre bancos de dados}

\section{Risk factors for neonatal mortality in Blumenau, Santa Catarina: linkage between database}

Ernani Tiaraju de Santa Helena 1

Clóvis Arlindo de Sousa 2

Cristiane Amorin da Silva 3

1 Departamento de Medicina. Universidade Regional de Blumenau. Rua Antônio da Veiga, 140. Blumenau, SC, Brasil. CEP: 89.010-971. E-mail: erntsh@furb.br

2,3 Residência Multiprofissional em Saúde da Família. Universidade Regional de Blumenau. Blumenau, SC, Brasil.

\begin{abstract}
Objectives : to determine neonatal mortality risk factors in the city of Blumenau, Santa Catarina using the variables reports in the Born Alive Forms.

Methods: this is a cohort study comprised of 3812 born alive children among which 26 neonatal deaths occurred. Risk factors were assessed through logistic regression.

Results: the final model disclosed significant association with neonatal for: newborns with birth weight under 2500 grams (OR=4.70; IC95\%: 1.31 16.87); pregnancy term under 36 weeks $(O R=4.16$; IC95\% 1.22-14.20); APGAR in the fifth minute following birth below eight $(O R=62.38$; IC95\%: 22.31-174.39) and presence of anomaly $(O R=63.19$; IC95\%: 15.17-263.15). Social and economic, medical and assistance and healthcare services did not show any significant association and couldn't be set apart from biological risk factors.

Conclusions: data indicate the value of identi fying principal risk factors for neonatal deaths, confirming the influence of biological factors strongly related to neonatal infantile mortality causes.

Key words Neonatal mortality, Risk factors, Alive born
\end{abstract}

\section{Resumo}

Objetivos: investigar os fatores de risco para a mortalidade neonatal no municipio de Blumenau, Santa Catarina, empregando como possíveis fatores de risco as variáveis apresentadas nas "Declarações de Nascidos Vivos".

Métodos: trata-se de um estudo de coorte com ap resentação de 3812 nascidos vivos, entre os quais ocorreram 26 óbitos neonatais. Os fatores de risco foram estimados utilizando a reg ressão logística.

Resultados: no modelo final apresentaram associ ação significativa com o óbito no periodo neonatal os nascidos com peso menor que 2500 gramas $(O R=4,70$ IC95\%: 1,31-16,87); menor que 36 semanas $\left(O R=4,16\right.$; IC95\%: 1,22-14,20); Apgar no $5^{\circ}$ minuto menor que oito $(O R=62,38$; IC95\%: 22,31-174,39) e presença de anomalia $(O R=63,19$ : IC95\%: 15,17263,15). As variáveis socioeconômicas, médico-assis tenciais e de serviços de saúde não apresentaram associação significativa, estando confundidas com os fatores de risco biológicos.

Conclusões: os dados mostram a importância de identificação dos principais fatores de risco de óbito para periodo neonatal, reafirmando a influência dos fatores biológicos fortemente relacionados ao componente neonatal da mortalidade infantil.

Palavras-chave Mortalidade neonatal, Fatores de risco, Nascidos vivos 


\section{Introdução}

No Brasil, os últimos 20 anos foram marcados por uma queda na mortalidade infantil, e embora tenham existido períodos de estabilidade e mesmo de elevação dos coeficientes, as maiores reduções de mortalidade infantil ocorrendo principalmente nas regiões Sul e Centro-Oeste.1,2 Estudos descrevem relevantes reduções nos estados do Ceará, São Paulo $^{3}$ e Rio Grande do Sul. 4 Em várias regiões do país a mortalidade neonatal (óbitos do nascimento até 27 dias completos de vida) se constitui no principal componente da mortalidade infantil, e o estudo dos seus possíveis fatores de risco cresce em importância. 5,6

A mortalidade neonatal é determinada, em geral, por malformações congênitas e, em nosso meio, por causas perinatais, como baixo peso ao nascer, prematuridade, problemas relacionados ao parto $\mathrm{e}$ pós-parto imediato, precariedade nos serviços de saúde de pré-natal e parto. Essas causas apontam para relações complexas existentes entre uma condição geral de vida e os padrões de práticas de saúde e acesso a serviços. 7,8

A maioria das causas dos óbitos neonatais é considerada passível de prevenção, através de condutas adequadas ao controle da gravidez e de atenção ao parto. ${ }^{9}$ Por isso, torna-se mister o monitoramento e entendimento dos fatores de risco para mortalidade neonatal. $\mathrm{O}$ estudo dos fatores de risco para mortalidade neonatal é útil: a) para identificar grupos expostos a diferentes variáveis de risco, e sua relação com a evolução para óbito; b) entender elementos da corrente de acontecimentos que tem relação com a mortalidade infantil neonatal; c) conhecer as necessidades para intervenções voltadas a mortalidade no período neonatal. 10

Houve nos últimos anos um aumento considerável de estudos relacionados aos riscos de mortalidade neonatal. Isso se deve, pelo menos em parte, à inclusão da "Declaração de Nascidos Vivos" (DNV) como documento obrigatório a ser preenchido nos serviços de saúde, e à melhoria do registro de óbitos de recém-nascidos pelo "Sistema de Informação de Mortalidade" (SIM).11,12

A descentralização do gerenciamento nos serviços de saúde dos municípios facilitou a formulação de ações de vigilância e estudos nos serviços, através da produção e acesso a banco de dados atualizados referentes à mortalidade, nascimentos $\mathrm{e}$ produção dos serviços de saúde. 13

Em Blumenau, a mortalidade infantil vem declinando desde 1996 e seu principal componente é a mortalidade neonatal. ${ }^{14} \mathrm{Em} \mathrm{1998}$, foi implantado um sistema de vigilância de recém-nascido considerado de risco (RN - Risco), definido pela combinação de riscos biológicos (baixo peso, prematuridade, tipo de gravidez, Apgar e anomalia) e risco social (escolaridade, estado civil, idade e ocupação materna) obtidos na DNV. Esse sistema de vigilância inclui visita às famílias das crianças de risco. No entanto, não se possui uma avaliação de quais variáveis de risco possuem maior valor preditor para mortalidade neonatal. 15

O objetivo deste estudo foi investigar as variáveis apresentadas nas "Declarações de Nascidos Vivos" (DNV) como fatores de risco para a mortalidade neonatal no município de Blumenau, Santa Catarina, em 2002.

\section{Métodos}

Este é um estudo de coorte baseado em dados vinculados da "Declaração de Nascidos Vivos" (DNV) do "Sistema de Informação sobre Nascidos Vivos" (SINASC), "Declaração de Óbitos" (DO) do "Sistema de Informação sobre Mortalidade" (SIM) no período de 2002, em Blumenau.

O Município de Blumenau possui uma área de $510,3 \mathrm{Km}^{2}$ e está localizado a leste do Estado de Santa Catarina, Brasil. Sua população rural diminuiu de 25,281 em 1991 para 19,865 em 2000. A população urbana aumentou de 185,875 para 241,943 no mesmo período. 16 É uma cidade de porte médio com população total em 2002 estimada de 272,283 habitantes. 17 Os principais serviços da região são as atividades têxteis, comércio e turismo.

A assistência hospitalar do município é formada por quatro hospitais, perfazendo 604 leitos, 60 laboratórios de análises clínicas, 192 clínicas médicas e 130 farmácias, no ano de 2000. No ano de 2003, a Fundação Hospitalar $\cong$ Hospital Santo Antonio, credenciado para atendimento à gestante de alto risco, dispunha de 149 leitos, o Hospital Santa Isabel possuía 249 leitos, o Hospital Santa Catarina possuia 160 leitos e o Hospital Misericórdia da Vila Itoupava, 47 leitos. Desses quatro, dois possuíam UTI pediátrica, o Hospital Santo Antonio e o Santa Catarina.

A implantação do Sistema de Informação de Nascidos Vivos em Blumenau teve início em 30 de setembro 1993. A partir de maio de 1998, todos os Nascidos Vivos de Risco passaram a ter suas visitas domiciliares registradas em uma "Ficha de Visita Domiciliar para Recém-Nascidos de Risco", permitindo uma maior atenção e registro das características que envolvem as crianças de risco e, obser- 
vando as representações por unidade de saúde. 18

A população estudada constituiu-se de 3950 nascidos vivos de mães residentes em Blumenau, entre 1 de janeiro de 2002 e 31 de dezembro de 2002. Considerou-se um nascimento vivo a definição estabelecida desde 1950 pela Organização Mundial de Saúde (OMS) a fim de padronizar conceitos e possibilitar a comparação internacional de dados:

"Nascido vivo é a expulsão ou extração completa do corpo da mãe, independente mente da duração da gravidez, de um produto de concepção que, depois da sepa ração, respire ou apresente qualquer outro sinal de vida, tal como batimentos do coração, pulsações do cordão umbilical ou movimentos efetivos dos músculos de contração voluntária, estando ou não cortado o cordão umbilical e estando ou não desprendida a placenta" (Laurenti e Mello J o rge; 2004: 65).19

Para critério de inclusão dos nascidos era necessário o retorno das fichas de visita domiciliar (pelas Unidades de Saúde) das crianças de risco das mães que possuíam como endereço de residência o município de Blumenau.

Realizou-se o procedimento de linkage 20 entre banco de dados de nascidos vivos $\cong$ SINASC (tomados como coorte estática), identificando-se os óbitos neonatais (menores de 28 dias), aqui tomados como variável de desfecho, obtidos da "Declaração de Óbito" ocorridos entre 1 de janeiro de 2002 e 27 de janeiro de 2003, registrados no "Sistema de Informação de Mortalidade". Estabeleceu-se ainda um segundo link como o banco de dados local das "Fichas de Visita Domiciliar para Recém-Nascido de Risco", para se obter as unidades de saúde que atenderam os recém-nascidos.

As variáveis de exposição foram agrupadas por blocos de risco: a) sócio-demográfico: idade da mãe (menor que 20 anos), escolaridade materna (ensino fundamental incompleto), estado civil da mãe (solteira); ocupação materna (dona de casa); b) assistência ambulatorial: consultas de pré-natal (menor ou igual a três), unidades de saúde Programa Saúde da Família (PSF), ambulatórios gerais (AGs) e unidades avançadas de saúde (UAS); c) assistência hospitalar: horário de nascimento (plantão); hospitais (com Unidade de Tratamento Intensivo), tipo de parto (cesáreo ou espontâneo); d) biológico: muito baixo peso ao nascer (menor de 2500 gramas); Apgar no $5^{\circ}$ minuto (menor que oito), duração da gestação (menor ou igual a 36 semanas), tipo de gravidez (dupla e mais); nascidos com anomalia; sexo (masculino).
Para análise dos dados, o arquivo final foi transferido para osoftware de análise estatística Stata 8.0. Realizou-se inicialmente uma análise para distribuição de freqüência, e o coeficiente de mortalidade neonatal para expressar a probabilidade de morrer até os 27 dias, foi obtido através da fórmula:

$\begin{gathered}\text { Óbito de crianças } \\ \begin{array}{c}\text { Coeficiente de } \\ \text { mortalidade } \\ \text { neonatal }\end{array}\end{gathered}=\frac{\begin{array}{c}\text { menores de } 28 \text { dias nascidas } \\ \text { em Blumenal em } 2002\end{array}}{\begin{array}{l}\text { Total de nascidos vivos } \\ \text { em Blumenau em } 2002\end{array}} \times 1000$

$\mathrm{Na}$ análise univariada, o odds ratio (OR) foi utilizado para verificar a associação entre as variáveis de exposição e o óbito neonatal com respectivos intervalos de confiança de $95 \%$ (IC95\%). O OR foi empregado como aproximação do risco relativo, dado o pequeno número de eventos e pela opção de uso da regressão logística não-condicional para o ajustamento dos potenciais efeitos de confusão. Para o cálculo da significância estatística da associação, utilizou-se o teste de Qui-Quadrado, sendo aceito um nível de significância para $p \leq 0,05$.

Foi construído um modelo hierarquizado agrupando as variáveis por bloco (sócio-demográfico, ambulatorial, hospitalar e biológico), para observar o ajustamento no interior do bloco. Para a composição do modelo final adotou-se o procedimento stepwise forward para avaliação de confundimento, iniciandose por aquele bloco de variáveis mais diretamente relacionado com o desfecho, cujas variáveis se apresentaram com maior OR e com $p \cong 0,2$ na análise univariada.

\section{Resultados}

Obteve-se uma coorte de 3812 recém-nascidos, com perda de $1,88 \%$, devido ao não retorno da ficha de visita domiciliar pelas Unidades de Saúde, erro de preenchimento de algumas características importantes à análise e mãe não residente no município de Blumenau.

Do total de nascidos vivos (3812) apresentaramse 1499 (39,32\%) como recém-nascidos de "risco". Dos 26 óbitos neonatais $22(84,6 \%)$ ocorreram no grupo dos neonatos de "risco" e $4(15,4 \%)$ entre os considerados "não-risco" (Tabela 1).

O "Coeficiente de Mortalidade Neonatal Total" apresentou-se 8,0 por mil nascidos vivos. Entre os nascidos considerados "não risco" foi de 1,7 por mil. Elevou-se substancialmente entre os neonatos de "risco" (17,7 por mil). 
Tabela 1

Freqüência dos grupos considerados de risco e Coeficiente de Mortalidade Neonatal por mil nascidos vivos. Blumenau, Santa Catarina, 2002.

\begin{tabular}{|c|c|c|c|c|c|c|}
\hline \multirow{2}{*}{ Grupos } & \multicolumn{2}{|c|}{ Óbitos } & \multicolumn{2}{|c|}{ Nascidos vivos } & \multirow{2}{*}{ Total } & \multirow{2}{*}{$\begin{array}{c}\text { Coeficiente } \\
\text { de mortalidade } \\
\text { neonatal }\end{array}$} \\
\hline & $(n)$ & $(\%)$ & (n) & $(\%)$ & & \\
\hline Risco* & 27 & 84,10 & 1499 & 39,32 & 1526 & 17,7 \\
\hline Não risco & 4 & 15,40 & 2313 & 60,68 & 2317 & 1,7 \\
\hline Total & 31 & 100,00 & 3812 & 100,00 & 3843 & 8,0 \\
\hline
\end{tabular}

$\star O R=10,64 ; \quad(I C 95 \% 3,71-30,49) ; p=0,001$.

\section{Tabela 2}

Fatores sócio-demográficos de risco para mortalidade neonatal. Blumenau, Santa Catarina, 2002.

\begin{tabular}{|c|c|c|c|c|c|c|}
\hline Variável & Casos & Controles & $\begin{array}{c}\text { Odds Ratio } \\
\text { (não ajustado) }\end{array}$ & IC95\% & $\begin{array}{l}\text { Odds Ratio } \\
\text { (ajustado) }\end{array}$ & IC95\% \\
\hline \multicolumn{7}{|l|}{ Escolaridade materna } \\
\hline $1^{\circ}$ grau completo & 23 & 3294 & 1 & & & \\
\hline $1^{\circ} \mathrm{grau}$ incompleto & 8 & 499 & $2,29 * *$ & $1,01-5,15$ & $2,60 *$ & $0,79-8,50$ \\
\hline \multicolumn{7}{|l|}{ Estado civil } \\
\hline Casada/União consensual & 13 & 2365 & 1 & & & \\
\hline Solteira & 18 & 1424 & $2,30 * *$ & $1,12-4,70$ & 2,19 & $0,64-7,47$ \\
\hline \multicolumn{7}{|l|}{ Idade materna } \\
\hline 20 anos & 24 & 3309 & 1 & & & \\
\hline Menos de 20 anos & 7 & 485 & $1,98 *$ & $0,85-4,64$ & 1,07 & $0,31-3,70$ \\
\hline \multicolumn{7}{|l|}{ Ocupação mãe } \\
\hline Trabalha fora/ocupada & 18 & 2279 & 1 & & & \\
\hline Dona de casa & 13 & 1515 & 1,08 & $0,53-2,22$ & - & - \\
\hline
\end{tabular}

$* p<0,20 ; * *<0,05$

\section{Tabela 3}

Fatores de risco assistencial ambulatorial para mortalidade neonatal. Blumenau, Santa Catarina, 2002.

\begin{tabular}{|c|c|c|c|c|c|c|}
\hline Variável & Casos & Controles & $\begin{array}{c}\text { Odds Ratio } \\
\text { (não ajustado) }\end{array}$ & $\mathrm{IC95 \%}$ & $\begin{array}{l}\text { Odds Ratio } \\
\text { (ajustado) }\end{array}$ & IC95\% \\
\hline \multicolumn{7}{|c|}{ Número consultas pré-natal } \\
\hline 3 e mais & 25 & 3689 & 1 & & & \\
\hline menor ou igual a 3 & 6 & 100 & $8,97 * * *$ & $3,60-22,37$ & 2,13 & $0,44-10,35$ \\
\hline \multicolumn{7}{|l|}{ Tipo de Unidade } \\
\hline Saúde da Família & 14 & 1490 & 1 & & & \\
\hline AGs e UASt & 17 & 2302 & 1,27 & $0,63-2,59$ & - & - \\
\hline
\end{tabular}

$* * * p<0,01 ;+A G=$ Ambulatório geral; UAS = Unidade avançada de saúde 
Fatores de risco assistenciais hospitalares para mortalidade neonatal. Blumenau, Santa Catarina, 2002.

\begin{tabular}{|c|c|c|c|c|c|c|}
\hline Variável & Casos & Controles & $\begin{array}{c}\text { Odds Ratio } \\
\text { (não ajustado) }\end{array}$ & IC95\% & $\begin{array}{l}\text { Odds Ratio } \\
\text { (ajustado) }\end{array}$ & IC95\% \\
\hline \multicolumn{7}{|l|}{ Hospital } \\
\hline Sem UTI & 3 & 1453 & 1 & & & \\
\hline Com UTI & 28 & 2336 & $5,82 * * *$ & $1,76-19,18$ & 0,85 & $0,19-3,81$ \\
\hline \multicolumn{7}{|l|}{ Hora nascimento } \\
\hline Diumo & 17 & 2443 & 1 & & & \\
\hline Plantão noturno & 13 & 1343 & 1,51 & $0,73-3,05$ & - & - \\
\hline \multicolumn{7}{|l|}{ Tipo de parto } \\
\hline Vaginal & 11 & 1761 & 1 & & & \\
\hline Cesáreo & 20 & 2033 & 1,57 * & $0,75-3,28$ & - & - \\
\hline
\end{tabular}

$* p<0,20 ; * * * p<0,01$

Tabela 5

Fatores de risco biológicos para mortalidade neonatal. Blumenau, Santa Catarina, 2002.

\begin{tabular}{|c|c|c|c|c|c|c|}
\hline Variável & Casos & Controles & $\begin{array}{c}\text { Odds Ratio } \\
\text { (não ajustado) }\end{array}$ & IC95\% & $\begin{array}{l}\text { Odds Ratio } \\
\text { (ajustado) }\end{array}$ & IC95\% \\
\hline \multicolumn{7}{|l|}{ Peso ao nascer } \\
\hline 2500 gramas e mais & 9 & 3538 & 1 & & & \\
\hline Menos de 2500 gramas & 22 & 255 & $34,06 * * *$ & $15,52-74,76$ & 4,70 ** & $1,31-16,87$ \\
\hline \multicolumn{7}{|l|}{ Prematuridade } \\
\hline 36 semanas e mais & 12 & 3585 & 1 & & & \\
\hline Menos de 36 semanas & 19 & 209 & $27,20 * * *$ & $13,03-56,80$ & 4,16 ** & $1,22-14,20$ \\
\hline \multicolumn{7}{|l|}{ Apgar $5^{\circ} \min$} \\
\hline 8 e mais & 9 & 3733 & 1 & & & \\
\hline menos de 8 & 22 & 57 & $160,08 * * *$ & $70,61-362,92$ & $62,38 * * *$ & $22,31-174,39$ \\
\hline \multicolumn{7}{|l|}{ Tipo de gravidez } \\
\hline Única & 26 & 3732 & 1 & & & \\
\hline Dupla e mais & 5 & 62 & $11,48 * * *$ & $4,26-30,88$ & $2,62 *$ & $0,49-13,93$ \\
\hline \multicolumn{7}{|l|}{ Anomalia } \\
\hline Sem anomalia & 23 & 3767 & 1 & & & \\
\hline Presença de anomalia & 8 & 24 & $54,20 * * *$ & $22,05-133,19$ & $63,19 * * *$ & $15,17-263,15$ \\
\hline
\end{tabular}

$* p<0,20 ; * * p<0,05 ; * * * p<0,01$ 
As Tabelas 2 a 5 apresentam os odds ratio univariados e ajustados das variáveis de estudo.

$\mathrm{Na}$ análise univariada, apresentaram associação com a mortalidade no período neonatal as seguintes variáveis: mães com escolaridade equivalente ao ensino fundamental incompleto ou menos apresentaram OR=2,29 (IC95\%: 1,01-5,15), mães com estado civil solteira $\mathrm{OR}=2,29$ (IC95\%: 1,12-4,70), consulta de pré-natal menor ou igual a três apresentou $\mathrm{OR}=8,97$ (IC95\%: 3,60-22,37), nascer em hospital com Unidade de Tratamento Intensivo (UTI) $\mathrm{OR}=5,82$ (IC95\%: 1,76-19,18), peso ao nascer menor que $2500 \mathrm{OR}=34,06$ (IC95\%: 15,52-74,76), prematuridade $\mathrm{OR}=27,20$ (IC95\%: 13,03-56,80), Apgar menor que oito OR=161,85 (IC95\%: 71,34367,19 ), gravidez dupla ou mais $\mathrm{OR}=11,48$ (IC95\%: 4,26-30,88) e presença de anomalia congênita $\mathrm{OR}=54,20$ (IC95\%: 22,05-133,19).

A análise das variáveis no através de regressão logística produziu um ajuste que manteve somente as seguintes variáveis do bloco de risco biológico: nascidos com peso menor que 2500 gramas $\mathrm{OR}=4,70$ (IC95\%: 1,31-16,87); prematuridade $\mathrm{OR}=4,16$ (IC95\%: 1,22-14,20), Apgar menor que oito OR=62,38 (IC95\%: 22,31-174,39) e presença de anomalia OR=63,19 (IC95\%: 15,18-263,15).

\section{Discussão}

Os estudos de coorte (em especial de nascidos vivos) ainda são considerados raros em Santa Catarina, mas se mostram viáveis como neste estudo, admitindo algumas considerações: o elevado percentual de nascimentos hospitalares (98\%) facilitou a realização do estudo; o tamanho da cidade estudada, que por não ser uma metrópole com altos fluxos migratórios, propiciou melhor controle dos eventos e a disponibilidade de um fluxo de informação padronizado de nascidos vivos, óbitos e um sistema de vigilância à criança de risco implantado na rede municipal.

A implantação dos sistemas nacionais de informação no SUS tem permitido um crescimento de estudos nos serviços de saúde, 12,21 contudo, dadas as grandes diferenças regionais no país, é necessário levar em conta a qualidade de preenchimento das "Declarações de Óbitos e de Nascidos Vivos".22 Em Blumenau, alguns estudos já vêm demonstrando a consistência das informações desses sistemas, posto que as informações das DNV são preenchidas por enfermeiras com dados obtidos dos pediatras que assistem as salas de parto e que preenchem por sua vez as DOs. $14,18,23$
O estudo da mortalidade neonatal pode auxiliar na avaliação da qualidade de vida de uma população, por refletir, ao menos em parte, a disponibilidade e a qualidade da assistência prestada nos serviços de saúde ambulatoriais e hospitalares.22,24

Em um município do Estado de São Paulo, no ano de 1997,15 a maior proporção de óbitos ocorreu no período neonatal $(66,1 \%)$, diminuindo à medida que as idades chegam a um ano. Em outro estudo de mortalidade em menores de um ano no sul do Brasil24 os óbitos foram concentrados na fase neonatal (77,3\%). Em uma metanálise referente aos períodos entre 1985-1987 e entre 1995-1997 sobre mortalidade infantil, 25 os autores constataram um crescimento na proporção de óbitos neonatais no Brasil de $46,4 \%$ para $59,2 \%$, com maior proporção na região Centro-Oeste (77,6\%) e menor na região Sul (59\%).

No México, ${ }^{26}$ propôs-se um volume de $50 \%$ de óbitos neonatais para a mortalidade infantil como indicador de que a região detém boas condições de saúde na infância. Entretanto, nesse período (19801990 ) o país tinha $48 \%$ de óbitos neonatais, mas um coeficiente de 15,2 óbitos neonatais por mil nascidos vivos.

O coeficiente de mortalidade neonatal observado em Blumenau ( 8,0 por mil) mostrou-se bem abaixo da média nacional. No ano de 2002, a mortalidade neonatal no Brasil foi de 12,6 óbitos por mil nascidos vivos. As regiões de maior mortalidade foram a Norte $(13,7)$ e Nordeste $(15,0)$, e as regiões Sul $(10,4)$, Sudeste $(11,5)$ e Centro-Oeste $(12,0)$ apresentaram menor mortalidade, com destaque para o Estado do Rio Grande do Sul $(9,5)$ e Santa Catarina $(9,9) 27,28$ com valores mais próximos aos óbitos neonatais apresentados em Blumenau.

Má qualidade de vida, precariedade do local de residência e a ausência do pai, são algumas das condições socioeconômicas que podem representar riscos ao neonato. ${ }^{29} \mathrm{Um}$ estudo realizado no Chile 30 demonstrou um risco quase cinco vezes maior de mortalidade neonatal para filhos de mãe sem instrução, atribuindo 35,7\% dos casos à desigualdade social. Em nosso estudo, a escolaridade e o estado civil da mãe se apresentaram associados ao risco neonatal com $\mathrm{OR} \cong 2$, tanto na análise univariada como após a regressão, mas perdendo significância estatística após o ajuste, o que pode ser explicado em parte pela força das variáveis biológicas.

A prematuridade, escore baixo do Apgar, peso baixo ao nascer e tipo de gravidez dupla e mais, são os fatores de risco descritos na literatura que apresentam maior associação com a mortalidade no período neonatal,4,13,24,25 o que também se mostra em Blumenau. Recém-nascidos com baixo peso são 
considerados mais vulneráveis a transtornos metabólicos e imaturidade pulmonar, podendo agravar alguns eventos que acometem os recémnascidos, aumentando as chances de mortalidade. ${ }^{2}$

As maiores prevalências de baixo peso encontram-se principalmente em países em desenvolvimento, o que é alvo de vários estudos epidemiológicos na tentativa de intervenções que possam reduzir sua ocorrência. $25,26,30$

Com relação à freqüência de baixo peso em Blumenau no período de 1994 a 1997, houve uma diminuição de $8,7 \%$ para $6,8 \%$, a prematuridade reduziu-se de $5,1 \%$ para $4,9 \%$ e a proporção de nascidos vivos com Apgar de $5^{\circ}$ minuto menor ou igual a sete, diminuiu consideralvelmente (de $6,5 \%$ para $3,1 \%) .18$

Entre as principais causas de baixo peso ao nascer encontram-se as infecções, a desnutrição materna e o tabagismo. Existem conseqüentemente dois mecanismos que conduzem o baixo peso ao nascer: o retardo de crescimento intra-uterino e a prematuridade. A prematuridade ocorre quando a criança nasce antes de completar 37 semanas de gestação. Já o retardo de crescimento intra-uterino acontece quando a criança nasce com peso abaixo do valor limite para sua idade gestacional, também conhecido como desnutrição fetal. 6

Em um estudo conduzido recentemente na Universidade do Alabama ${ }^{29}$ o peso corporal e o Apgar baixos foram apontados como os maiores preditores de mortalidade neonatal. Outro estudo realizado em por Morais Neto e Barros, ${ }^{2}$ em Goiânia, encontrou razões de odds significantes em baixo peso ao nascer $(\mathrm{OR}=27,60$; IC95\%: 20,40$37,33)$ e gestação inferior a 37 semanas $(\mathrm{OR}=37,68$; IC95\%: 28,35-50,06).

Os autores citados acima ponderam que o peso ao nascer e a duração da gestação não devem ser estudados como fatores de risco isolados, mas como mediadores que atuam em diversos determinantes e condicionantes da mortalidade infantil, como por exemplo: características socioeconômicas da mãe, escolaridade, características biológicas, hábitos de vida, acesso aos serviços de saúde. Estas características devem ser consideradas como marcadores para predizer os riscos de mortalidade neonatal. Consideram ainda que os nascidos vivos de gravidez múltipla apresentam uma alta incidência de baixo peso ao nascer e prematuridade. Por essa razão, os gemelares são excluídos da maioria dos estudos sobre fatores de risco para mortalidade infantil. No presente estudo, o efeito da gemelariadade é ajustado no modelo final, perdendo significância pela força e relacionamento com baixo peso e prematuridade.
O tipo de parto cesáreo não se apresentou como risco para óbito neonatal. Alguns autores colocam seu fator protetor mesmo depois de controlar os efeitos das variáveis de confusão. ${ }^{2}$ No entanto, não é consensual seu efeito protetor para a mortalidade neonatal.2,31 Com relação aos nascidos vivos em hospitais privados, o efeito protetor do parto cesáreo pode estar conexo com: acesso ao serviço de saúde, atenção ao parto, nível socioeconômico entre outros. Um estudo realizado no Brasil apresenta a relação entre o nível socioeconômico e o tipo de parto cesáreo. 31

Não se observou influência do horário de nascimento sobre a mortalidade neonatal, como observado em um estudo realizado na Suécia, 28 onde foi encontrado um alto risco de morte neonatal em nascimentos no período noturno (plantão). Em nascimentos prematuros, o horário da noite em relação ao período diurno, também apresentou alto risco em países como Alemanha e México. 26,32

Com relação as variáveis assistenciais (ambulatoriais e hospitalares), os dados para a assistência ambulatorial expressam que os neonatos com baixo número de consultas de pré-natal apresentaram associação com mortalidade na análise univariada, ocorrendo perda de significância após o ajuste no modelo final.

Os hospitais com unidade de tratamento intensivo pediátrica (UTI) apresentaram associação na análise univariada com a mortalidade neonatal, que foi expurgada no modelo final. Essa associação talvez se devesse ao direcionamento de gestantes de risco para hospitais com esse tipo de serviço e menos a problemas de qualidade de assistência ao parto.

No modelo final, a presença de anomalia permaneceu com elevada associação para a mortalidade neonatal, praticamente sem sofrer ajuste, representando um possível caminho explicativo diferente em relação aos principais fatores de risco biológicos (prematuridade, Apgar baixo, tipo de gravidez e peso ao nascer muito baixo), que talvez se expressem como causa de óbito no grupo das causas perinatais.

Com a determinação das políticas para a promoção de saúde da mulher antes da concepção, durante a gestação e, da criança depois do parto, pode-se promover um desenvolvimento contínuo e integrado à saúde, com objetivo de garantir acesso aos serviços de qualidade. Esse avanço pode ser decisivo no combate a mortalidade neonatal. No entanto, vem sendo acentuada a desigualdade nas sociedades em que o acesso a esse tipo de assistência não está garantido, como é o caso do Brasil, onde não se conseguiu implantar concretamente um sistema de saúde realmente universal e eqüitativo. 22 
No município de Blumenau, a Secretaria de Saúde tem realizado esforços direcionados à proteção e promoção de saúde de sua população. Em 2003 foi lançado o "Projeto Nascer com Saúde", com a justificativa de garantir qualidade na assistência prestada, com ênfase ao acesso integral à saúde, a

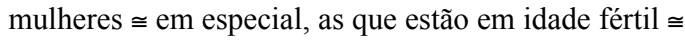
e crianças até cinco anos de idade. A grande intenção do projeto era promover a saúde materno-infantil e reduzir e morbimortalidade materna e de recémnascidos até cinco anos de vida.

As estratégias envolviam múltiplas dimensões, desde o processo de planejamento da concepção até o crescimento e desenvolvimento do ser humano, tendo como objetivo a qualidade da atenção, enfatizando a acessibilidade e utilização dos serviços de saúde locais e regionais e amplitudes das ações, envolvendo desde o contexto individual até o comunitário, interdisciplinaridade, controle social e a intersetorialidade.

Uma boa parte dos óbitos neonatais podem ser evitados por medidas de acompanhamento do trabalho de parto e assistência imediata do pós-parto. ${ }^{2} \mathrm{O}$ esforço na melhoria deve envolver não somente o acesso à assistência pré-natal de qualidade, mas também medidas de continuidade de assistência até o momento do parto e a assistência hospitalar adequada. ${ }^{11,25}$

Em Blumenau, a implantação do Sistema de Vigilância ao Recém-Nascido de Risco, apesar de demonstrar boa sensibilidade e especificidade para óbito infantil e hospitalização, precisa ser aprimorado em relação às ações que visam a redução dos óbitos neonatais. ${ }^{23} \mathrm{O}$ presente estudo fornece subsídios para o sistema de vigilância ao apontar as variáveis com maior poder explicativo, que podem ser alvo de atuação preventiva.

A elaboração de estudos em serviço que utilizam a forma de "linkage" entre bancos de dados de nascidos vivos e óbitos neonatais mostra viabilidade e grandes vantagens por permitir uma avaliação de consistência das bases de dados oficiais e auxiliar no monitoramento e a avaliação das ações preventivas da rede pública. 14,20

\section{Agradecimentos}

Agradecemos a Prof $f^{a}$. Dra. Hillegonda Maria Dutilh Novaes, do Departamento de Medicina Preventiva(,) da Faculdade de Medicina da Universidade de São Paulo, pelas sugestões e críticas na confecção final do artigo.

\section{Referências}

1. Waldman EA. A transição epidemiológica: tendências e diferenciais dos padrões de morbimortalidade em diferentes regiões do mundo. Mundo Saúde 2000; 24: 10-8.

2. Morais Neto OL, Barros MBA. Fatores de risco para mortalidade neonatal e pós-neonatal na Região CentroOeste do Brasil: linkage entre banco de dados de nascidos vivos e óbitos infantis. Cad Saúde Pública 2000; 16: 477-85.

3. Victora CG, Barros FC. Infant mortality due to perinatal causes in Brazil: trends, regional patterns and possible interventions. São Paulo Med J 2001; 119: 33-42.

4. Victora CG, Barros FC, Tomasi E, Menezes AM, Horta BL, Weiderpass E, César JA, Costa JSD, Olinto MT, Halpern R, Garcia MM, Vaughan JP. Tendências e diferenciais na saúde materno-infantil: delineamento e metodologia das coortes de 1982 e 1993 de mães e crianças de Pelotas, Rio Grande do Sul. Cad Saúde Pública 1996; 12: 7-14.

5. Monteiro CA, Pino HP, Benicio MHA, Victora CG. Better prospects for child surviral. World Health Forum 1989; 10: $222-7$.

6. Almeida MF, Mello Jorge MP. Pequenos para idade gestacional: fator de risco para mortalidade neonatal. Rev Saúde Pública 1998; 32: 217- 24

7. Victora CG, Vaughn JP, Barros FC. Explaining trends in inequities: evidence from Brazilian child health studies. Lancet 2000; 356: 1093- 8 . 
8. Machado CJ, Hill K. Determinants of neonatal and postneonatal mortality in the City of São Paulo. Rev Bras Epidemiol 2003; 6: 345-58.

9. Victora CG, Grassi PR, Schmidt AM. Situação de saúde da criança em área da região Sul do Brasil, 1980-1992: tendências temporais e distribuição espacial. Rev Saúde Pública 1994; 28: 423- 32.

10. César, CLG. Fatores de risco associados à mortalidade infantil em duas áreas da região metropolitana de São Paulo (Brasil), 1984-1985: proposta de instrumentos preditivos. Rev Saúde Pública 1990; 24: 300-10.

11. Barros FC, Victora CG, Vaughan JP. The pelotas bitrh cohort study, 1982-1987: strategies for following up 6000 children in a developing country. Perinatal Pediatr Epidemiíol 1990; 4: 267-82.

12. Carvalho DM. Grandes sistemas nacionais de informação em saúde: revisão e discussão da situação atual. IESUS 1997; VI: 7-46.

13. Almeida MF, Novaes HMD, Alencar GP, Rodrigues LC. Mortalidade neonatal no Município de São Paulo: influência do peso ao nascer e de fatores sócio-demográficos e assistências. Rev Bras Epidemiol 2002; 5: 93- 106

14. Santa Helena ET, Rosa MB. Avaliação da qualidade das informações relativas aos óbitos em menores de um ano em Blumenau, 1998. Rev Bras Saúde Matern Infant 2003; 3: $75-83$.

15. Gomes JO, Santo AH. Mortalidade infantil em município da região Centro-Oeste Paulista, Brasil, 1990 a 1992. Rev Saúde Pública 1997; 31: 330-41.

16. IPEA (Instituto de Pesquisa Econômica Aplicada), Fundação João Pinheiro. Programa das Nações Unidas para o Desenvolvimento. Atlas de desenvolvimento humano no Brasil [programa de computador]. Versão 1.0.1. Rio de Janeiro; 2003.

17. Ministério da Saúde. IBGE (Instituto Brasileiro de Geografia e Estatísticao). Indicadores municipais de saúde. Cad Inf Saúde. Portal da Saúde [on line]. 2004. Disponível em URL: http://www.datasus.gov.br [2004 ago 20].

18. Santa Helena ET, Wisbeck J. Implantação do SINASC e perfil dos nascidos vivos de Blumenau, 1994-1997. IESUS 1998; VII: 36-42.

19. Laurenty R, Mello Jorge MHP. O atestado de óbito. 5. ed. São Paulo: Centro colaborador da OMS para a família de classificações, Centro Brasileiro de Classificação de Doenças; 2004. (Divulgação. n. 1).

20. Almeida MF, Mello Jorge MHP. O uso da técnica de linkage de sistemas de informação em estudos de coorte sobre mortalidade neonatal. Rev Saúde Pública 1996; 30: 141-7.
21. Carvalho AO, Paula Eduardo MB. Sistemas de informação em saúde para os municípios. São Paulo: Faculdade de Saúde Pública da Universidade de São Paulo; 1998. (Série Saúde \& Cidadania).

22. Lansky S, França E, Leal MC. Mortalidade perinatal e evitabilidade: revisão da literatura. Rev Saúde Pública 2002; 36: 759- 72 .

23. Santa Helena ETS, Wisbeck J, Bottos MA. RNRISCO $\cong$ Recém-nascido de risco em Blumenau: avaliação do sistema de vigilância e uso de linkage entre SINASC/SIM/ RNRISCO. In: Anais do VI Congresso Brasileiro de Saúde Coletiva; 200028 agosto - 1 setembro; Salvador, Brasil. Cienc Saúde Colet 2000; 5 ( Supl): 354-5.

24. Souza RKT, Gotlieb SLD. Probabilidade de morrer no primeiro ano de vida em área urbana da região Sul, Brasil. Rev Saúde Pública 1993; 27: 445-54

25. Victora CG, Barros FC. Infanty mortality due to perinatal causes in Brazil: trends, regional patterns and possible interventions. São Paulo Med J 2001; 119: 33-42.

26. Vandale SBA, Ramon AR, Kageyama ML. Time-trends and cause of infant, neonatal and postneonatal mortality in México, 1980-1990. S alud Pública Mex 1997; 39: 48-52.

27. Ministério da Saúde. Departamento de Análise da Situação de Saúde. Sistema de Vigilância em Saúde. Sistema de Informações sobre mortalidade - SIM [on line]. 2002. Disponível em URL: http://www.datasus.gov.br [2004 set 26].

28. Ministério da Saúde. Sistema de Vigilância em Saúde. Departamento de Análise da Situação de Saúde. Sistema de Informações sobre Nascidos Vivos - SINASC [on line]. 2002. Disponível em URL: http://www.datasus.gov.br [2004 set 26]

29. Luo ZC, Kalberg J. Timing of birth and infant early neonatal mortality in Sweden 1973-95: longitudinal birth register study. Br Med J 2001; 323: 1327-30.

30. Hollstein RD, Veja J, Carvajal Y. Desiguladades sociales e salud. Nível socioeconomico y mortalidad infantil Chile, 1985-1995. Rev Med Chile 1998; 126: 333-40.

31. Ambalavanan N, Carlo WA. Comparison of the prediction of extremely low birth weight neonatal mortality by regression analysis and by neural networks. Early Hum Dev 2001; 65: 123-37.

32. Faúndes A, Cecatti JG. A operação cesária no Brasil Incidência, tendências, causas, conseqüências e propostas de ação. Cad Saúde Pública 1991; 7: 150-73.

Recebido em 10 de se tembro de 2004

Versão final em 24 de janeiro de 2005

Aprovado em 21 de fevereiro de 2005 